\title{
Harmonically-related gratings-based quadrature phase interferometers
}

Zahid Yaqoob, Jigang Wu, Xiquan Cui, Xin Heng, Changhuei Yang

Zahid Yaqoob, Jigang Wu, Xiquan Cui, Xin Heng, Changhuei Yang, "Harmonically-related gratings-based quadrature phase interferometers," Proc. SPIE 6488, Practical Holography XXI: Materials and Applications, 64880J (20 February 2007); doi: 10.1117/12.701585

Event: Integrated Optoelectronic Devices 2007, 2007, San Jose, California, United States 


\title{
Harmonically-related gratings-based quadrature phase interferometers
}

\author{
Zahid Yaqoob, Jigang Wu, Xiquan Cui, Xin Heng, and Changhuei Yang \\ Biophotonics Laboratory, Department of Electrical Engineering \\ California Institute of Technology, Pasadena, CA 91125 \\ Email: zyaqoob@caltech.edu
}

\begin{abstract}
We report a new method for obtaining non-trivial phase difference between the output ports of an interferometer through the use of shallow diffraction gratings. We show that as opposed to a single shallow diffraction grating-based interferometer (which provides only trivial phase shifts, i.e., $0^{\circ}$ or $180^{\circ}$ ), a pair of harmonically-related shallow diffraction gratings can be used to design interferometers with non-trivial phase shifts between different output ports. More importantly, the phase shifts can be adjusted by simply shearing one grating with respect to the other. This approach does not change the path length relationships of the different interference beams within the interferometer, which is an advantage for metrology and low coherence interferometry applications.
\end{abstract}

Keywords: Diffraction gratings, quadrature phase interferometry, metrology, medical and biological imaging

\section{INTRODUCTION}

Optical phase imaging is a powerful technique, which uses the differences in phase of light that is transmitted through or reflected off a sample to yield contrasting intensity images of the sample under study. Features such as nanometerscale resolution and non-contact mode operation make this technique extremely useful for imaging of thin, transparent, loosely bound, or easily damaged samples. Quantitative phase measurements are desired in numerous applications in cell biology and medical research [1] as well as metrology [2]. For instance, the knowledge of the shifts in the phase of light can be used to obtain quantitative information about the morphology and dynamics of living cells without staining.

Traditional phase-contrast imaging techniques such as Zernike phase [3,4] and Nomarski differential interference contrast (DIC) [5] render excellent phase contrast images; however, the phase information is only qualitative in nature. Phase-shifting interferometry [6-8], a method for quantitative phase imaging (QPI), generally requires recording of four interferograms with precise $\pi / 2$ phase shifts of the reference field. Other techniques for QPI include digital holography [9] and Hilbert phase microscopy [10,11], which depend on recording of high frequency spatial fringes for successful phase unwrapping. In addition, polarization-based quadrature phase interferometers have also been reported [12]. It is to be noted that these non-trivial encoding schemes (time, space and polarization) add complexity to the system or make the phase retrieval process computationally intensive.

We note that multiport fiber-based systems such as $3 \times 3$ couplers provide non-trivial phase difference between different ports, which can be manipulated for instantaneous quadrature phase measurements [13, 14]. Such fiber-based techniques are simple and direct; however, they are only single-point measurement techniques and thus require lateral scanning of the sample beam to obtain two-dimensional phase images of the sample under study. A free-space equivalent of a $3 \times 3$ fiber-optic is desirable as it will allow instantaneous full-field quadrature phase measurements, suitable for spatially-resolved investigations of biological samples. In this paper, we report a new method for obtaining non-trivial phase difference between different output ports of an interferometer through the use of shallow diffraction gratings. It is shown that a single shallow diffraction grating-based interferometers provide only trivial phase shifts between different output ports. On the contrary, a pair of harmonically-related shallow diffraction gratings can be used to realize a interferometers where the phase shifts between different output ports can be simply adjusted by shearing one

Practical Holography XXI: Materials and Applications, edited by Roger A. Lessard, Hans I. Bjelkhagen, Proc. of SPIE Vol. 6488, 64880J, (2007) · 0277-786X/07/\$18 · doi: 10.1117/12.701585 
grating with respect to the other. We note that the reported method to obtain non-trivial phase shift is simple and direct, and opens new possibilities for full-field quadrature phase interferometry [15].

\section{PHASE OF DIFFRACTED LIGHT IN SHALLOW GRATINGS}

Consider a sinusoidal phase grating as shown in Fig. 1. The complex transmittance of a sinusoidal phase grating can be expressed as:

$$
t(x)=\sum_{m=-\infty}^{\infty} J_{m}(\alpha) \exp \left\{j m\left(\frac{2 \pi}{\Lambda} x+\xi\left(x_{o}\right)+\frac{\pi}{2}\right)\right\}
$$

where $\alpha, \Lambda$, and $x_{o}$ are the amplitude of phase modulation, period, and displacement from the origin along $x$-direction, respectively, of the phase grating. Moreover, $J_{m}(\alpha)$ is $m^{\text {th }}$ order Bessel function of the first kind and $\xi\left(x_{o}\right)=2 \pi x_{o} / \Lambda$. Using the identity $J_{-m}(\alpha)=(-1)^{m} J_{m}(\alpha)$, the relative phase of the $m^{\text {th }}$ diffracted order with respect to the zeroth order is given by:

$$
\phi\left(x_{o}\right)=\left\{\begin{array}{cc}
m\left\{\xi\left(x_{o}\right)+\frac{\pi}{2}\right\}, & m \geq 1 \\
|m|\left\{-\xi\left(x_{o}\right)+\frac{\pi}{2}\right\} . & m<1
\end{array}\right.
$$

Equation (2) shows that there exists an additional non-trivial phase shift associated with each diffraction order. Moreover, the additional phase shift can be simply adjusted by shifting the phase grating along the $x$-direction.

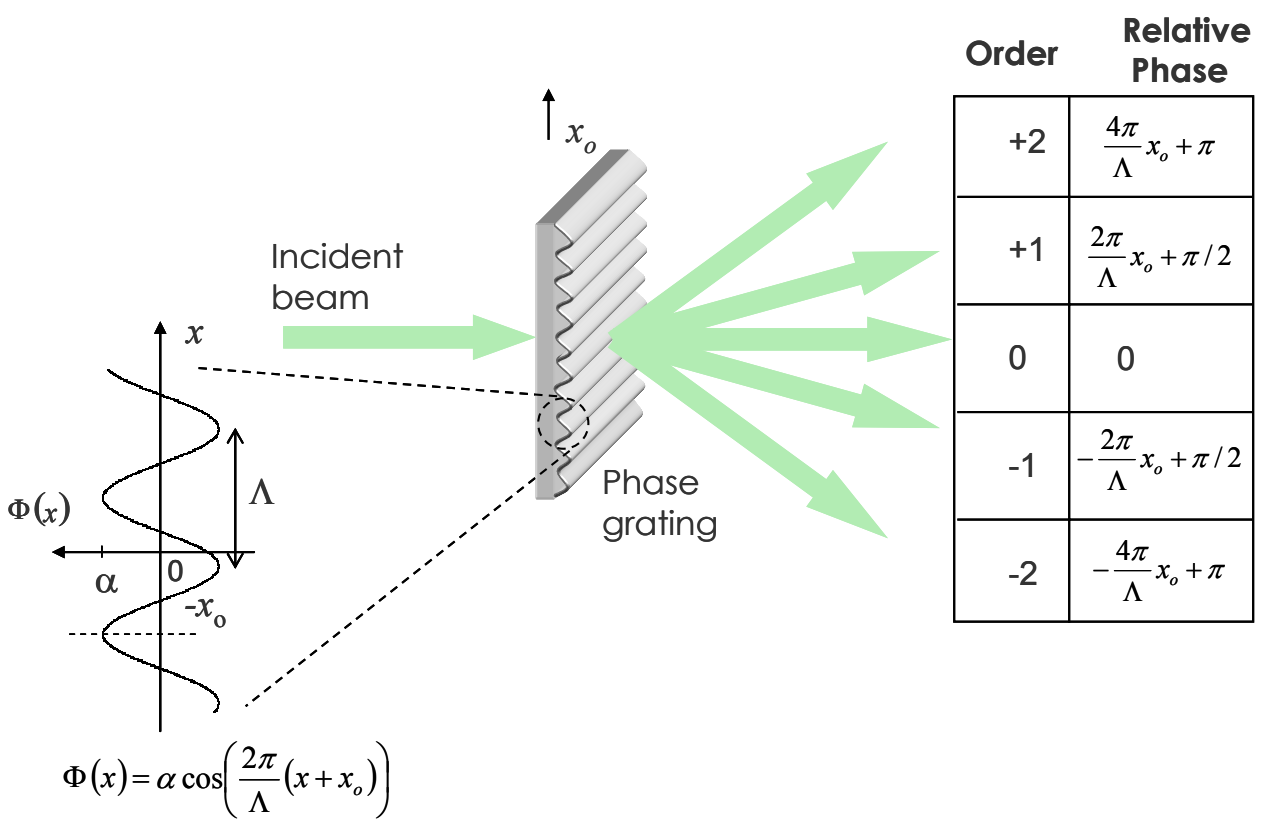

Figure 1: Spatial phase modulation in a sinusoidal phase grating. The diffraction orders have additional nontrivial phase shifts which can be adjusted by shifting the phase grating along $x$-direction. 


\section{SINGLE GRATING-BASED INTERFEROMETERS}

Although the diffracted light in diffraction gratings acquires a unique phase with respect to the undiffracted light, the phase shifts between different output ports of single grating-based Michelson / Mach-Zehnder interferometers are only trivial in nature. To better understand this phenomenon and the operation of our harmonically-related gratings-based interferometer, we start by examining a simpler system - a shallow phase grating-based beam combiner such as in a Mach Zehnder interferometer [see Fig. 2(a)].

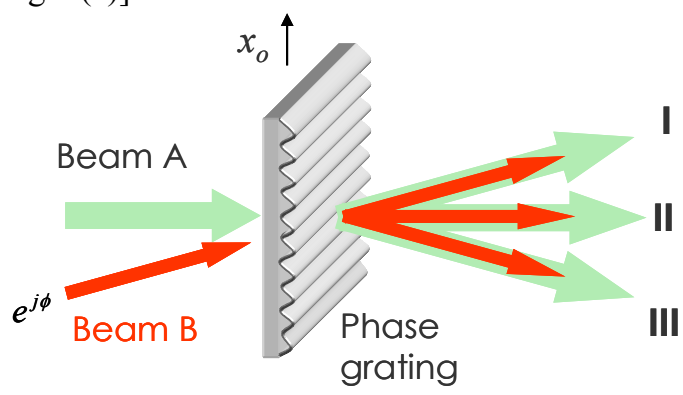

(a)

\begin{tabular}{|c|c|c|c|c|c|c|}
\hline \multirow{2}{*}{$\begin{array}{c}\text { Output } \\
\text { Ports }\end{array}$} & \multicolumn{2}{|c|}{ Beam A } & \multicolumn{2}{|c|}{ Beam B } & \multirow{2}{*}{\multicolumn{2}{|c|}{$\begin{array}{l}\text { Interference } \\
\text { Signals }\end{array}$}} \\
\hline & Order & Phase & Order & Phase & & \\
\hline I & +1 & $\frac{2 \pi}{\Lambda} x_{o}+\pi / 2$ & 0 & $\phi$ & $\propto \cos$ & {$\left[\frac{2 \pi}{\Lambda} x_{o}+\frac{\pi}{2}-\phi\right.$} \\
\hline II & 0 & 0 & -1 & $\phi-\frac{2 \pi}{\Lambda} x_{o}+\pi / 2$ & $\propto \cos$ & {$\left[\frac{2 \pi}{\Lambda} x_{o}-\frac{\pi}{2}-\phi\right.$} \\
\hline III & -1 & $-\frac{2 \pi}{\Lambda} x_{o}+\pi / 2$ & -2 & $\phi-\frac{4 \pi}{\Lambda} x_{o}+\pi$ & $\propto \cos$ & {$\left[\frac{2 \pi}{\Lambda} x_{o}-\frac{\pi}{2}-\phi\right.$} \\
\hline
\end{tabular}

(b)

Figure 2: (a) Single sinusoidal phase grating as a beam combiner. Beams A and B diffract and combine at the output ports I, II, and III. (b) Diffraction orders associated with beams A and B and corresponding additional nontrivial phase shifts. Note that the interference signals at the three output ports have trivial phase shifts.

As shown in Fig. 2(a), a shallow phase grating combines two incident beams, i.e., beams A and B at the output ports I, II, and III. The corresponding diffraction orders and associated additional non-trivial phase shifts are shown in Fig. 2(b). Although the diffracted beams acquire a unique non-trivial phase shift, the interference terms in Fig. 2(b) indicate that the phase shifts between different output ports of single grating-based combiner (or a Mach-Zehnder interferometer) are only trivial in nature.

Similarly, in a single shallow diffraction grating-based Michelson interferometer arrangement shown in Fig. 3(a), the diffracted (reference) beam acquires additional non-trivial phase shift with respect to the undiffracted (sample) beam. The returning sample and reference beams undergo a second diffraction and combine at the three output ports I, II, and III of the interferometer. The overall phase information of the coincident beams, shown as dashed black and red lines [see Fig. 3], at the output ports can be used to write the interference terms as:

$$
\begin{array}{ll}
\text { Interference signal at port I: } & i_{\mathrm{I}} \propto \cos \left\{2 k\left(d_{2}-d_{1}\right)+\frac{4 \pi}{\Lambda} x_{o}\right\} \\
\text { Interference signal at port II: } & i_{\mathrm{II}} \propto \cos \left\{2 k\left(d_{2}-d_{1}\right)+\frac{4 \pi}{\Lambda} x_{o}+\pi\right\} \\
\text { Interference signal at port III: } & i_{\mathrm{III}} \propto \cos \left\{2 k\left(d_{2}-d_{1}\right)+\frac{4 \pi}{\Lambda} x_{o}+\pi\right\}
\end{array}
$$




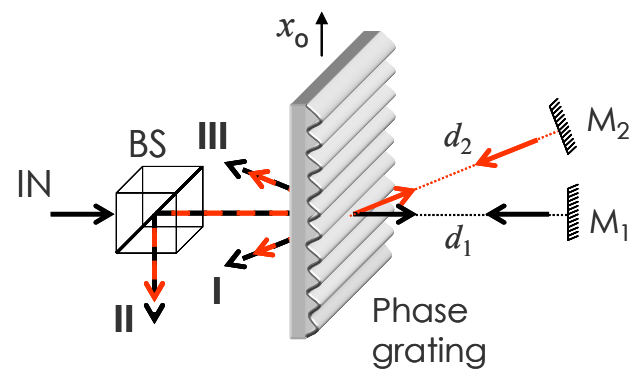

Figure 3: Schematic of a Michelson interferometer using a single shallow diffraction grating. Dashed black and red lines are representative of coincident sample and reference beam at output ports I, II, and III of the interferometer. $\mathrm{M}_{i}$ : $i$ th Mirror; BS: Beam splitter.

Equation (3) indicates that the ports II and III of a single shallow grating-based Michelson interferometer are in phase whereas the port I of the interferometer is $180^{\circ}$ out of phase with respect to the other two output ports. This shows that a single grating-based interferometer is unsuitable for extracting quadrature signals.

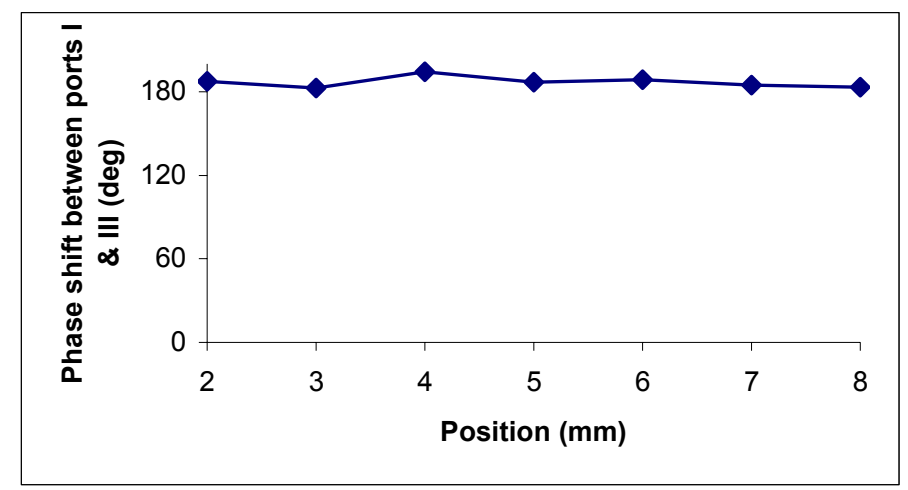

(a)

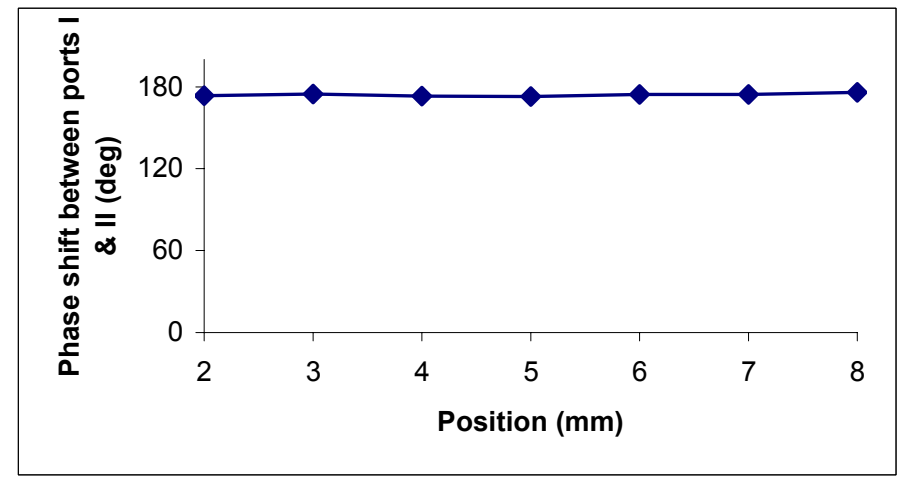

(b)

Figure 4: Measured phase shifts between (a) ports I \& III and (b) ports I \& II of a single grating-based Michelson interferometer versus grating displacement along the $x$-direction.

To confirm the above argument, an experimental setup as shown in Fig. 3 was realized using a HeNe laser $(\lambda=633 \mathrm{~nm})$ and a shallow 600 grooves $/ \mathrm{mm}$ blazed transmission grating (Thorlabs, Inc., GT25-06V). The reference mirror was mounted on a voice coil to modulate the reference arm. Heterodyne interference signals were acquired at the three 
output ports using New Focus photodetectors (model 2001). The grating was translated along the $x$-direction in order to measure the phase shifts between different ports of the interferometer for various positions of the grating. For each position of the grating, the acquired heterodyne signals at the three ports were analyzed to determine the phase shifts between the three output ports. Figure 4 (a) and (b) show phase shifts between ports I \& III and ports I \& II, respectively; the experimental data verifies that for a single grating-based Michelson setup ports II and III are in phase whereas port I is $\sim 180^{\circ}$ out of phase with respect to the ports II and III, indicating that a single grating-based setup is inappropriate for quadrature interferometry.

\section{HARMONICALLY-RELATED GRATING PAIR-BASED INTERFEROMETERS}

As shown in the previous section, a non-trivial phase shift is conferred on the interference term associated with each output port of a single-grating based interferometer. However, since the amount of non-trivial phase shift is the same for each output port, the configurations shown in Figs. 2 and 3 yield only trivial phase shift between the output ports. Fortunately, this effect does not extend to interferometers that contain two or more shallow gratings. In this section, we show that Mach Zehnder and Michelson interferometers based on two harmonically-related shallow diffraction gratings can be used to acquire quadrature signals that give direct access to sample phase information.

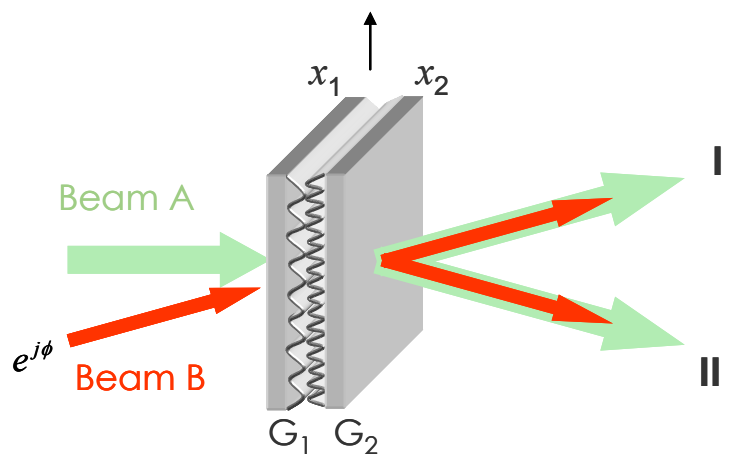

(a)

\begin{tabular}{c|c|c|c|c|c|}
$\begin{array}{c}\text { Output } \\
\text { Ports }\end{array}$ & \multicolumn{2}{c}{$\begin{array}{c}\text { Order } \\
\text { Oram A }\end{array}$} & \multicolumn{2}{c}{$\begin{array}{c}\text { Order } \\
\text { Interference } \\
\text { Signals }\end{array}$} \\
\hline I & +1 & $\frac{2 \pi}{\Lambda_{1}} x_{1}+\pi / 2$ & 0 & $\phi$ & $\propto \cos \left[\frac{2 \pi}{\Lambda_{1}} x_{1}+\frac{\pi}{2}-\phi\right]$ \\
\hline II & -1 & $-\frac{2 \pi}{\Lambda_{1}} x_{1}+\pi / 2$ & -1 & $\phi-\frac{2 \pi}{\Lambda_{2}} x_{2}+\pi / 2$ & $\propto \cos \left[\frac{2 \pi}{\Lambda_{2}} x_{2}-\frac{2 \pi}{\Lambda_{1}} x_{1}-\phi\right]$ \\
\hline
\end{tabular}

(b)

Figure 5: (a) Harmonically-related phase grating pair as a beam combiner. Beams A and B combine at the output ports I and II. (b) Diffraction orders associated with beams A and B and corresponding additional non-trivial phase shifts. Interference signals at the three output ports have non-trivial phase shifts.

Consider a pair of harmonically-related grating pair as a final beam combiner in a Mach Zehnder setup [see Fig. 5(a)]. The parameters $x_{1}$ and $x_{2}$ represent the position of the two gratings with respect to the incident beams. Beam A is incident at normal to the grating pair. The angle of incidence of the second beam B is adjusted on the grating pair such that its 0 th order is coincident with the +1 order of beam A. Figure 5 (b) shows the diffraction orders as well as additional phase shifts of the beams arriving at ports I and II of the setup in Fig. 5(a). Figure 5(b) also shows the interference terms associated with the two output ports. We note that in the case of harmonically-related gratings pair 
based beam combiner, the phase shift between the output ports is not trivial. In fact, the phase is dependent on the relative position of the two gratings and is given by:

$$
\Delta \phi=\frac{2 \pi}{\Lambda_{2}}\left(x_{2}-x_{1}\right)-\frac{\pi}{2}
$$

To see whether the above findings also hold for a Michelson setup as well, we analyze the schematic shown in Fig. 6. We choose the period of first grating as twice the period of the second grating. The two gratings $\mathrm{G}_{1}$ and $\mathrm{G}_{2}$ are placed at distance $d_{1}$ and aligned such that the grating planes as well as grating vectors are parallel to that of each other. Two mirrors $M_{1}$ and $M_{2}$ are introduced in the setup and aligned such that the first order diffracted beams from $G_{1}$ meet with the undiffracted beam at $G_{2}$. The grating $G_{2}$ combines the two first order beams from $G_{1}$ to form the reference beam (dashed red-green line) as shown in Fig. 6. The undiffracted beam from $G_{1}$ passes straight through $G_{2}$ to form the sample arm of the interferometer.

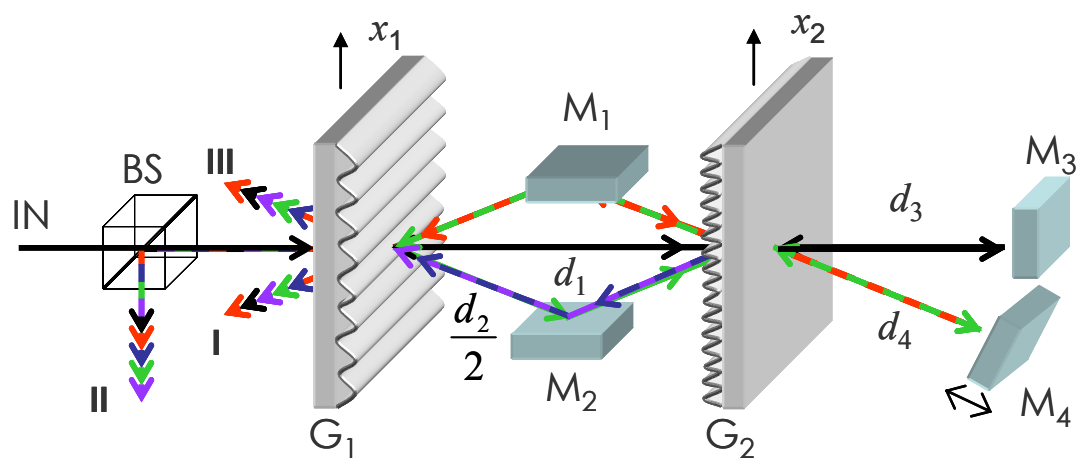

Figure 6: Modified Michelson interferometer design based on two harmonically-related shallow transmission gratings, where $d_{1}$ is the inter-grating distance. Parameters $x_{1}$ and $x_{2}$ correspond to the actuations of grating $\mathrm{G}_{1}$ and $\mathrm{G}_{2}$, respectively, whereas $d_{3}$ and $d_{4}$ represent the path lengths of the sample and reference arms, respectively. $\mathrm{M}_{i}$ : ith Mirror; BS: Beam splitter.

The returning sample beam arrives straight at $G_{1}$ whereas the reference arm beam reaches $G_{1}$ through the two possible paths set by the grating $G_{2}$ and mirrors $M_{1}$ and $M_{2}$. Next, the grating $G_{1}$ splits and combines the incoming sample and reference beams at ports I, II, and III of the interferometer; the coincident five field components are represented by a five-colored dashed line at each output port. The interference terms at the three output ports can be written in simplified form as:

$$
\begin{array}{ll}
\text { At port I: } & \propto \cos \left[2 k\left(d_{4}-d_{3}+d_{2}-d_{1}\right)+\phi^{\prime}\left(x_{1}, x_{2}\right)\right] \\
\text { At port II: } & \propto \cos \left[2 k\left(d_{4}-d_{3}+d_{2}-d_{1}\right)+\phi^{\prime \prime}\left(x_{1}, x_{2}\right)\right] \\
\text { At port III: } & \propto \cos \left[2 k\left(d_{4}-d_{3}+d_{2}-d_{1}\right)+\phi^{\prime \prime \prime}\left(x_{1}, x_{2}\right)\right]
\end{array}
$$

In Eq. (5), the only phase term that involves the path lengths $d_{i}, i=1,2,3,4$ is $2 k\left(d_{4}-d_{3}+d_{2}-d_{1}\right)$, and is common to all the three interference signals. As such, it yields only a trivial phase between different output ports. The non-trivial phase terms, which play a role in providing a non-trivial phase shift between the output ports, are $\phi^{\prime}\left(x_{1}, x_{2}\right), \phi^{\prime \prime}\left(x_{1}, x_{2}\right)$, and $\phi^{\prime \prime \prime}\left(x_{1}, x_{2}\right)$. These phase terms depend on the strength of the individual field components arriving at the output ports [16]. Moreover, the phase terms do not depend on the path lengths and can be solely adjusted by shearing of the harmonically-related gratings with respect to each other.

For experimental verification, we realized a setup (shown in Fig. 6) using $633 \mathrm{~nm}$ HeNe laser. We chose $\mathrm{G}_{1}$ and $\mathrm{G}_{2}$ to be 600 grooves $/ \mathrm{mm}$ and 1200 grooves $/ \mathrm{mm}$, respectively, blazed transmission gratings. The path length $d_{1}$ between the gratings $\mathrm{G}_{1}$ and $\mathrm{G}_{2}$ was $\sim 20 \mathrm{~cm}$. The reference mirror $\mathrm{M}_{4}$ was mounted on a voice coil to modulate the reference arm. The grating $\mathrm{G}_{2}$ was mounted on a piezo actuator $(25.5 \mathrm{~nm} / \mathrm{V})$ for shearing along the $x$-direction. The heterodyne 
interference signals were acquired at the three output ports using the high-speed photodetectors and processed to determine the phase shift between different output ports versus the position of grating $\mathrm{G}_{2}$.

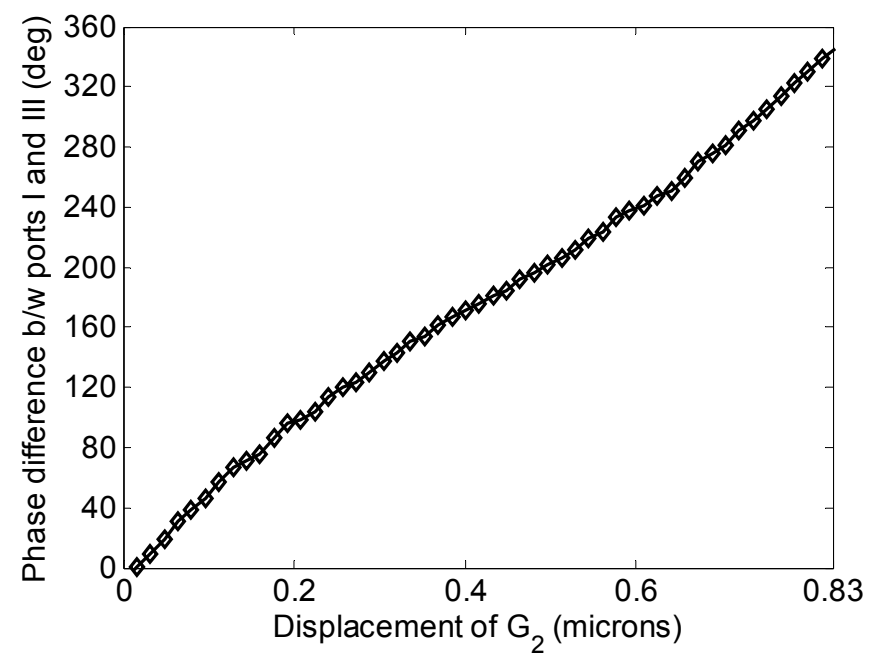

Figure 7: Measured phase shifts between ports I \& III of harmonically-related gratings-based Michelson interferometer versus relative position of grating $\mathrm{G}_{2}$ with respect to $\mathrm{G}_{1}$.

Figure 7 shows the measured phase shift between output ports I and III of the interferometer versus shearing of grating $\mathrm{G}_{2}$ up to one grating period. It can be seen that phase shift between ports I and III changes almost in a linear fashion as the grating G2 is sheared along the $x_{2}$ direction. This ability to achieve non-trivial phase shifts illustrates the possibility to design gratings-based interferometer for full-field quadrature phase interferometry. We note that this experiment does require gratings that are uniform and harmonically-related over the area of the incident beams.

\section{CONCLUSION}

In summary, we have proposed and experimentally demonstrated the use of harmonically-related shallow phase gratings to realize free-space equivalent of a $3 \times 3$ fiber-optic coupler. The non-trivial phase shift is adjusted by simply shearing the gratings with respect to each other. The ability to adjust the phase shifts between different ports of the interferometer is a useful feature of the design for quadrature phase interferometry. The main advantage of the proposed method for non-trivial phase is that the shearing of the gratings does not change the path length relationship between interference beams at the output ports of the interferometer, which is an advantage for metrology as well as low coherence interferometry applications. Note that a single shallow diffraction grating-based Michelson interferometer cannot provide but trivial phase shifts between different output ports; hence, it is not suitable for quadrature phase measurements. An additional advantage of the proposed method is that the harmonically-related gratings can also be fabricated or holographically recorded on a single substrate, making it possible to design compact imaging systems for full-field quadrature interferometry. Finally, this concept can be, in principle, extended to realize homodyne X-ray interferometers for quadrature phase measurements.

\section{REFERENCES}

[1] K. F. A. Ross, Phase contrast and interference microscopy for cell biologists. New York: St. Marin's Press, 1967.

[2] J. C. Shaw, "Metrology using differential phase-contrast microscopy," Microelectronic Engineering, vol. 13, pp. 527-530, 1991.

[3] F. Zernike, "Phase contrast, a new method for the microsopic observation of transparent objects," Physica, vol. 9, pp. 686-698, 1942.

[4] F. Zernike, "Phase contrast, a new method for the microscopic observation of transparent objects Part II," Physica, vol. 9, pp. 974-986, 1942. 
[5] G. Nomarski and A. R. Weill, "Application à la métallographie des méthodes interférentielles à deux ondes polarisées," Rev. Metall., vol. 2, pp. 121-128, 1955.

[6] Y. Y. Cheng and J. C. Wyant, "2-wavelength phase-shifting interferometry," Applied Optics, vol. 23, pp. 45394543, 1984.

[7] P. Hariharan and M. Roy, "Achromatic phase-shifting for two-wavelength phase-stepping interferometry," Optics Communications, vol. 126, pp. 220-222, 1996.

[8] W. Shimada, T. Sato, and T. Yatagai, "Optical surface microtopography using phase-shifting Nomarski microscope," Proc. SPIE vol. 1332, pp. 525-529, 1990.

[9] E. Cuche, F. Bevilacqua, and C. Depeursinge, "Digital holography for quantitative phase-contrast imaging," Optics Letters, vol. 24, pp. 291-293, 1999.

[10] T. Ikeda, G. Popescu, R. R. Dasari, and M. S. Feld, "Hilbert phase microscopy for investigating fast dynamics in transparent systems," Optics Letters, vol. 30, pp. 1165-1167, 2005.

[11] G. Popescu, T. Ikeda, C. A. Best, K. Badizadegan, R. R. Dasari, and M. S. Feld, "Erythrocyte structure and dynamics quantified by Hilbert phase microscopy," Journal of Biomedical Optics, vol. 10, 2005.

[12] D. S. Marx and D. Psaltis, "Polarization quadrature measurement of subwavelength diffracting structures," Applied Optics, vol. 36, pp. 6434-6440, 1997.

[13] M. A. Choma, "Instantaneous quadrature low-coherence interferometry with 3 x 3 fiber-optic couplers," Optics Letters, vol. 28, pp. 2162 - 2164, 2003.

[14] Z. Yaqoob, J. Fingler, X. Heng, and C. H. Yang, "Homodyne en face optical coherence tomography," Optics Letters, vol. 31, pp. 1815-1817, 2006.

[15] J. Wu, Z. Yaqoob, X. Cui, X. Heng, and C. Yang, "Three-dimensional imaging using grating-based quadrature phase interferometer," in conference on Three-Dimensional and Multidimensional Microscopy: Image Acquisition and Processing XIV (BO126), Paper No. 6443-17, SPIE symposium on Biomedical Optics, Photonics West 2007, San Jose, USA, January 20-25, 2007.

[16] Z. Yaqoob, J. Wu, X. Cui, X. Heng, and C. Yang, "Harmonically-related diffraction gratings-based interferometer for quadrature phase measurements," submitted for publication in Optics Express, 2006. 